\title{
Avaliação de I 66 ETEs em operação no país, COMPREENDENDO DIVERSAS TECNOLOGIAS. PARTE I - ANÁLISE de DESEMPENHO
}

\section{EVALUATION OF I 66 TREATMENT PLANTS OPERATING IN BRAZIL, COMPRISING SEVERAL TECHNOlOgIES. PART I - PERFORMANCE ANALYSIS}

\author{
SÍlVIA M. A. CORRÊA OLIVEIRA
}

Engenheira eletricista. Mestre em Saneamento, Meio Ambiente e Recursos Hídricos pela UFMG. Doutoranda na área de Saneamento na UFMG

\begin{abstract}
MARCOS VON SPERLING
Doutor em Engenharia Ambiental pelo Imperial College, Universidade de Londres. Professor Adjunto do Departamento de Engenharia Sanitária e Ambiental (DESA) da UFMG
\end{abstract}

Recebido: 20/01/05 Aceito: 20/10/05

\section{RESUMO}

O trabalho apresenta uma análise de desempenho de 166 estações de tratamento de esgotos urbanos em operação nos estados de Minas Gerais e de São Paulo. Foram investigadas seis modalidades de tratamento diferentes, compreendendo os processos: (a) fossa séptica seguida de filtro anaeróbio, (b) lagoas facultativas, (c) lagoas anaeróbias seguidas por lagoas facultativas, (d) lodos ativados, (e) reatores UASB operando isoladamente e (f) reatores UASB seguidos por pós-tratamento. A avaliação considerou as concentrações efluentes e as eficiências de remoção dos constituintes DBO, DQO, SST, NTK, PT e coliformes (organismos termotolerantes), que foram comparadas com valores típicos reportados na literatura técnica. Em geral, o desempenho na remoção de matéria orgânica esteve dentro do esperado. As eficiências de remoção de SST estiveram abaixo, enquanto a remoção de coliformes foi maior que a descrita na literatura. A remoção de nutrientes foi baixa, já que nenhuma das modalidades analisadas foi projetada com este objetivo.

PALAVRAS-CHAVE: Qualidade de efluente, avaliação de desempenho, tratamento de esgotos domésticos.

\begin{abstract}
The paper analyses the performance of 166 wastewater treatment plants operating in Brazil. Six different treatment technologies have been investigated, comprising the following processes: septic tank + anaerobic filter, facultative pond, anaerobic pond + facultative pond, activated sludge, UASB reactors alone, UASB reactors followed by post-treatment. The objective of the research was to evaluate the effluent quality and the removal efficiencies of the parameters $B O D$, COD, TSS, coliforms, TKN and phosphorus. The paper compares the effluent quality and the removal efficiencies with typical values reported by the technical literature. In general, the performance in the removal of organic matter was as expected. TSS removal efficiencies were lower than reported, but coliform removal was higher than described in the literature. Nutrient removal was low, but none of the treatment processes have been designed with this objective.
\end{abstract}

KEYWORDS: Effluent quality, performance evaluation, wastewater treatment.

\section{INTRODUÇÃO}

O impacto do lançamento de efluentes originados de estações de tratamento de esgotos em corpos d'água é motivo de grande preocupação para a maioria dos países. Uma série de legislações ambientais, critérios, políticas e revisões procuram influir tanto na seleção dos locais de descarga quanto no nível de tratamento exigido para garantir que os impactos ambientais provocados pela disposição destes efluentes tratados sejam aceitáveis.

Vários autores (Dean \& Forsythe, 1976a, 1976b; Niku et al, 1981;
Berthouex e Hunter, 1983; Vaughan e Russel, 1983; Smith et al, 2001) publicaram estudos sobre a verificação do atendimento aos padrôes de lançamento e sobre desempenho de processos de tratamento, considerando a qualidade dos efluentes. Todos estes trabalhos procuraram dar subsídios às agências ambientais para uma definição adequada dos padróes de lançamento e padróes dos corpos d'água. No Brasil, apesar de estudos e avaliaçôes em escala piloto ou em ETEs individuais (sumariadas em Andrade Neto, 1997; von Sperling, 1998; Campos, 1999; Chernicharo, 2001; von Sperling e Chernicharo, 2000, 2002), o conhecimento sobre o desempenho das tecnologias de tratamento de esgotos em operação no país é relativamente esparso, havendo poucas consolidações estruturadas em termos de uma avaliação global.

Neste sentido, este trabalho é inédito e de grande relevância para a área de tratamento de esgotos, já que avalia o comportamento de 166 ETEs em operação no país, fornecendo informações reais do desempenho de seis processos de tratamento, em termos da qualidade do efluente gerado e da eficiência de remoção alcançada.

$\mathrm{Na}$ pesquisa foram utilizados os dados coletados no âmbito do projeto 
URBAGUA, estudo contratado pela FINEP, de interesse para a Agência Nacional de Águas (ANA), executado pelas unidades de pesquisa da Universidade de São Paulo e da Universidade Federal de Minas Gerais. Este projeto, de caráter técnico e em nível nacional, foi proposto para a criação de instrumentos para a gestão integrada da água em áreas urbanas, para fornecer subsídios ao Programa Nacional de Despoluição das Bacias Hidrográficas (PRODES) e como um estudo exploratório de um programa nacional de apoio à gestão.

As tecnologias de tratamento, mais usualmente utilizadas no Brasil, foram descritas e avaliadas, de forma comparativa, buscando a identificação de sistemas que apresentassem melhor desempenho. Para isto, os resultados de concentrações efluentes e eficiência de remoção dos constituintes DBO, DQO, SST (sólidos suspensos totais), NTK (nitrogênio total Kjeldahl), PT (fósforo total) e CF (coliformes fecais, ou termotolerantes) foram comparados com dados de desempenho esperado publicados pela literatura especializada. As tecnologias de tratamento selecionadas para estudo foram: fossa séptica seguida de filtro anaeróbio (FS+FA), lagoas facultativas (LF), lagoas anaeróbias seguidas por lagoas facultativas (LAN+LF), lodos ativados (LA), reatores UASB sem pós-tratamento (UASB), reatores UASB seguidos de pós-tratamento (UASB+POS).

\section{METODOLOGIA}

Como o projeto foi desenvolvido em parceria entre a USP e a UFMG, foram coletados dados de ETEs em operação nos estados de São Paulo e de Minas Gerais. As séries históricas de dados de monitoramento das ETEs, utilizadas no trabalho, foram obtidas através de entidades estaduais de controle ambiental, de prestadores de serviços de saneamento, estaduais e municipais, e de projetos de pesquisas da UFMG.

$O$ período de abrangência dos dados experimentais de monitoramento das ETEs variou de janeiro de 1995 a agosto de 2003 e foram analisados através de estudos estatísticos preliminares, visando o conhecimento e caracterização dos diversos sistemas de tratamento. A média aritmética, a mediana e os valores máximos e mínimos dos dados relativos aos constituintes DBO, DQO, SST, NTK, PT e CF foram calculados, assim como as eficiências de remoção das cargas poluidoras afluentes.

\section{RESULTADOS E DISCUSSÃO}

\section{Estatísticas descritivas}

Na Tabela 1 são apresentados os nomes das entidades que disponibilizaram os dados nos dois estados e o número de ETEs contidas em cada modalidade de tratamento.

As médias das concentrações afluentes e efluentes e das eficiências de remoção das diversas ETEs, relativas a estas seis tecnologias de tratamento, considerando os seis constituintes, são apresentadas na Tabela 2 .
Pela observação da Tabela 2, percebeu-se uma diferença bastante acentua$\mathrm{da}$, tanto nas concentraçôes afluentes e efluentes, quanto nas eficiências de remoção das diversas modalidades de tratamento, considerando todos os constituintes, como comentado detalhadamente a seguir.

FS+FA. O esgoto afluente à modalidade FS+FA mostrou-se bem mais concentrado que o usual para esgotos domésticos, considerando os constituintes DBO, DQO e SST. As concentraçôes efluentes médias de DBO, DQO e NTK foram maiores que as observadas para todas as outras modalidades de tratamento.

LF. As concentrações afluentes e efluentes médias observadas para as lagoas facultativas (LF) foram, também, bastante elevadas e acima dos valores considerados usuais para DBO, DQO e SST, como será discutido adiante. As eficiências médias de remoção de sólidos mostraram-se bem abaixo das observadas para as outras modalidades, devido, provavelmente, à presença de algas no efluente.

LAN+LF. Apesar do esgoto afluente aos sistemas australianos (LAN+LF) mostrar-se muito concentrado, a concentração efluente média de DBO observada para a modalidade foi bem menor que as verificadas para as LF (com concentrações afluentes parecidas) e para os reatores UASB (com concentraçôes afluentes menores). As eficiências médias de remoção dos constituintes $\mathrm{DBO}, \mathrm{DQO}$ e CF foram bastante expressivas e consideradas superiores ao esperado para este tipo de tratamento, como será comentado em itens adiante.

Tabela I - Origem dos dados de monitoramento das ETEs

\begin{tabular}{|c|c|c|c|c|c|c|c|c|}
\hline \multirow[t]{2}{*}{ Tipo de entidade } & \multirow[t]{2}{*}{ Nome } & \multicolumn{6}{|c|}{ Número de ETEs, por modalidade de tratamento } & \multirow{2}{*}{$\begin{array}{c}\mathrm{N}^{\circ} \\
\text { Total }\end{array}$} \\
\hline & & $\mathrm{FS}+\mathrm{FA}$ & $\mathrm{LF}$ & $\mathrm{LAN}+\mathrm{LF}$ & $\mathrm{LA}^{(1)}$ & UASB & $\mathrm{UASB}_{+} \mathrm{POS}^{(2)}$ & \\
\hline \multicolumn{9}{|c|}{ Minas Gerais } \\
\hline Cia. Estadual de Saneamento & COPASA & 2 & 2 & 1 & 6 & 4 & 3 & 19 \\
\hline Órgão ambiental & FEAM & 1 & 1 & 2 & & 2 & 1 & 6 \\
\hline Prestador de serviço municipal & Diversos & & & & & 2 & 2 & 4 \\
\hline Órgão de pesquisa & UFMG & & & & & 2 & 2 & 4 \\
\hline Total de ETEs em MG & & 3 & 3 & 3 & 6 & 10 & 8 & 33 \\
\hline \multicolumn{9}{|c|}{ São Paulo } \\
\hline Cia. Estadual de Saneamento & SABESP & 16 & 70 & 40 & 5 & & & 131 \\
\hline Prestador de serviço municipal & Diversos & & & & 2 & & & 2 \\
\hline Total de ETEs em SP & & 16 & 70 & 40 & 7 & 0 & 0 & 133 \\
\hline Total geral de ETEs & & 19 & 73 & 43 & 13 & 10 & 8 & 166 \\
\hline
\end{tabular}

(1) LA incluiu as modalidades convencional e aeração prolongada;

(2) UASB+POS incluiu os pós-tratamentos: filtro aerado (FAE); filtro anaeróbio (FA); filtro biológico percolador (FBP); flotação (FLOT); lagoas facultativas (LF) e lagoas de polimento (LP). 
Tabela 2 - Concentrações e eficiências médias de remoção dos diversos constituintes

\begin{tabular}{|c|c|c|c|c|c|c|c|c|}
\hline & Constituinte & Unidade & $\mathrm{FS}+\mathrm{FA}$ & $\mathrm{LF}$ & $\mathrm{LAN}+\mathrm{LF}$ & LA & UASB & $\mathrm{UASB}+\mathrm{POS}$ \\
\hline \multirow[t]{3}{*}{$\mathrm{DBO}$} & Afluente & $(\mathrm{mg} / \mathrm{L})$ & 665 & 553 & 510 & 315 & 371 & 362 \\
\hline & Efluente & $(\mathrm{mg} / \mathrm{L})$ & 292 & 136 & 89 & 35 & 98 & 42 \\
\hline & Eficiência remoção & $(\%)$ & 59 & 75 & 82 & 85 & 72 & 88 \\
\hline \multirow[t]{3}{*}{ DQO } & Afluente & $(\mathrm{mg} / \mathrm{L})$ & 1398 & 1187 & 1095 & 575 & 715 & 713 \\
\hline & Efluente & $(\mathrm{mg} / \mathrm{L})$ & 730 & 525 & 309 & 92 & 251 & 141 \\
\hline & Eficiência remoção & $(\%)$ & 51 & 55 & 71 & 81 & 59 & 77 \\
\hline \multirow[t]{3}{*}{ SST } & Afluente & $(\mathrm{mg} / \mathrm{L})$ & 479 & 430 & 411 & 252 & 289 & 334 \\
\hline & Efluente & $(\mathrm{mg} / \mathrm{L})$ & 165 & 216 & 153 & 57 & 85 & 51 \\
\hline & Eficiência remoção & $(\%)$ & 66 & 48 & 62 & 76 & 67 & 82 \\
\hline \multirow[t]{3}{*}{ NTK } & Afluente & $(\mathrm{mg} / \mathrm{L})$ & 78 & 69 & 78 & 47 & 43 & \\
\hline & Efluente & $(\mathrm{mg} / \mathrm{L})$ & 61 & 38 & 45 & 22 & 48 & \\
\hline & Eficiência remoção & $(\%)$ & 24 & 44 & 39 & 50 & -13 & 24 \\
\hline \multirow[t]{3}{*}{ PT } & Afluente & $(\mathrm{mg} / \mathrm{L})$ & 9 & 9 & 11 & 3 & 7 & 7 \\
\hline & Efluente & $(\mathrm{mg} / \mathrm{L})$ & 7 & 4 & 7 & 1 & 6 & 5 \\
\hline & Eficiência remoção & $(\%)$ & 30 & 46 & 36 & 46 & -1 & 23 \\
\hline \multirow[t]{3}{*}{$\mathrm{CF}\left({ }^{*}\right)$} & Afluente & $($ org/100mL) & $2,6 \times 10^{7}$ & $5,3 \times 10^{7}$ & $2,0 \times 10^{8}$ & $3,7 \times 10^{7}$ & $1,2 \times 10^{8}$ & $1,8 \times 10^{8}$ \\
\hline & Efluente & $(\operatorname{org} / 100 \mathrm{~mL})$ & $5,3 \times 10^{6}$ & $1,2 \times 10^{6}$ & $4,3 \times 10^{5}$ & $1,3 \times 10^{5}$ & $3,4 \times 10^{7}$ & $9,7 \times 10^{6}$ \\
\hline & Eficiência remoção & Unidades log & 0,9 & 1,6 & 2,2 & 2,0 & 0,6 & 2,8 \\
\hline
\end{tabular}

(*) Para coliformes, os valores referem-se à média geométrica das concentraçōes.

LA. Os sistemas de lodos ativados (LA) mostraram um bom desempenho, considerando as concentrações efluentes e as eficiências de remoção de praticamente todos os constituintes, mesmo considerando que as concentraçôes afluentes foram as menores observadas.

UASB. Os reatores UASB operando isoladamente apresentaram desempenhos razoáveis e condizentes com o esperado para a modalidade.

$\mathrm{UASB}+\mathrm{POS}$. Os resultados mostrados pelos reatores UASB, quando seguidos de pós-tratamento (UASB+POS) foram superiores, em alguns casos, aos obtidos pelos lodos ativados. Esses bons desempenhos foram observados na remoção de DBO, SST e CF.

Uma comparação mais detalhada das concentraçôes e eficiências observadas e os valores considerados típicos pela literatura foi efetuada para verificação do percentual de ETEs com resultados inferiores ou superiores às faixas reportadas. Esses resultados serão apresentados nos itens que se seguem.

Outras informaçôes relativas às concentraçōes afluentes e efluentes observadas para as seis modalidades de tratamento são apresentadas na forma de gráficos Box-whisker, conforme pode ser visto na
Figura 1. Nesses gráficos são representados os valores mínimos e máximos, os percentis 25 e $75 \%$ e a mediana das concentrações relativas aos constituintes DBO, DQO, SST, NTK, PT e CF.

Pela observação da Figura 1, notase que, em geral, as modalidades de tratamento apresentaram uma grande variabilidade nas concentraçôes afluentes e efluentes, considerando todos os constituintes analisados. A heterogeneidade dos dados disponibilizados pelas ETEs, mesmo dentro de uma mesma tipologia, foi expressiva e observada em todos os casos. Esta variação foi mais acentuada para a modalidade fossa-filtro (FS+FA), considerando principalmente as concentraçóes efluentes de DBO, DQO e SST. No entanto, para todas as tecnologias de tratamento, foram observados episódios esparsos de concentraçóes afluentes e efluentes muito abaixo ou acima da média observada.

\section{Comparação entre as concentrações afluentes observadas e reportadas na literatura}

A Tabela 3 apresenta uma comparação entre as concentrações afluentes re- portadas na literatura e as obtidas no presente estudo. Os valores de concentrações afluentes considerados típicos para esgotos brutos, predominantemente domésticos, foram determinados de acordo com faixas apresentadas por von Sperling (2005) e a composição da faixa de concentrações efetivamente observadas em escala real considerou os percentis de $10 \%$ e $90 \%$.

Observou-se que, de uma maneira geral, os esgotos afluentes apresentaram uma concentração média mais elevada do que usualmente expresso na literatura.

Algumas possibilidades aventadas para justificar o grande número de ETEs que apresentaram concentrações de DBO acima dos valores usuais seriam contribuições industriais não relatadas pelas estaçóes de tratamento ou o tipo de amostragem praticado. No caso de despejos industriais predominantemente orgânicos, as concentraçōes de DBO são usualmente mais elevadas do que as observadas em esgotos domésticos, o mesmo acontecendo quando se utilizam amostras simples, coletadas em horários de pico. Outras explicações possíveis seriam referentes ao baixo consumo per capita de água e, ainda, à existência de menores coeficientes de retorno. No entanto, se- 
Tabela 3 - Comparação entre concentrações afluentes usuais e concentrações médias reais dos constituintes

\begin{tabular}{|c|c|c|c|c|c|}
\hline \multirow[t]{2}{*}{ Constituinte } & \multicolumn{2}{|c|}{$\begin{array}{l}\text { Concentraçóes usuais } \\
\text { reportadas na literatura } \\
{ }^{(1)}\end{array}$} & \multicolumn{2}{|c|}{$\begin{array}{c}\text { Concentrações médias } \\
\text { observadas }\end{array}$} & \multirow[t]{2}{*}{$\begin{array}{c}\% \text { de ETEs fora } \\
\text { da faixa usual }\end{array}$} \\
\hline & Faixa & Típicas & Faixa $^{(2)}$ & Média & \\
\hline $\mathrm{DBO}(\mathrm{mg} / \mathrm{L})$ & $200-500$ & 350 & $284-804$ & 527 & 51 \\
\hline DQO $(\mathrm{mg} / \mathrm{L})$ & $400-800$ & 700 & $505-1616$ & 1113 & 86 \\
\hline SST (mg/L) & $200-450$ & 400 & $202-527$ & 435 & 42 \\
\hline $\mathrm{NT}^{(3)}(\mathrm{mg} / \mathrm{L})$ & $35-70$ & 50 & $39-84$ & 66 & 44 \\
\hline $\mathrm{PT}(\mathrm{mg} / \mathrm{L})$ & $4-15$ & 7 & $2-14$ & 8 & 25 \\
\hline CF (org/100mL) & $10^{6}-10^{9}$ & - & $1,3 \times 10^{7}-1,8 \times 10^{8}$ & $9,4 \times 10^{7}$ & 1 \\
\hline
\end{tabular}

(1) Adaptado de von Sperling, 2005

(2) Foram utilizados os percentis $10 \%$ (valor mínimo) e $90 \%$ (valor máximo) para composição das faixas reais

(3) Foram utilizados valores de NTK e NT

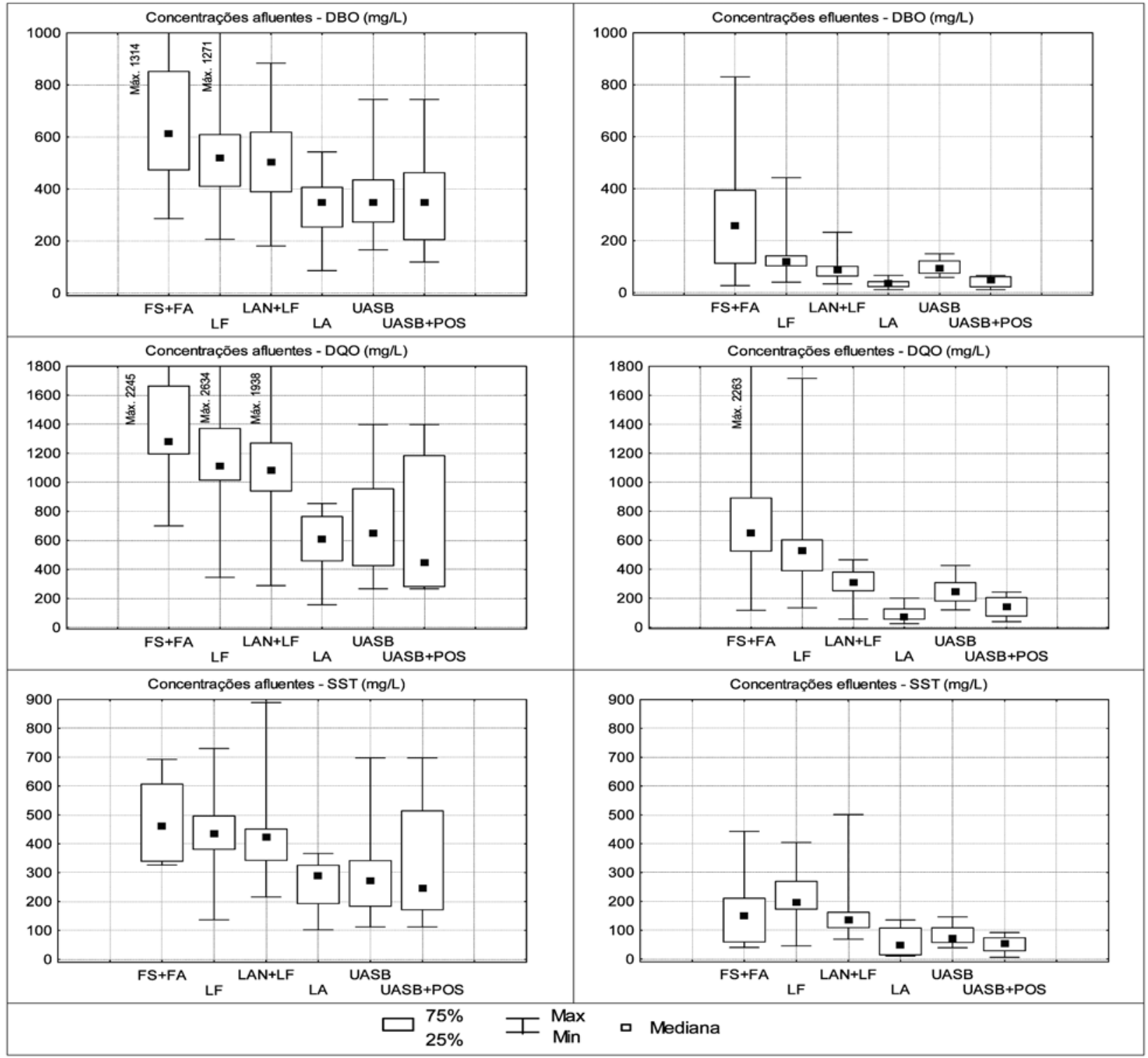

Figura I - Concentrações médias afluentes e efluentes dos diversos constituintes 


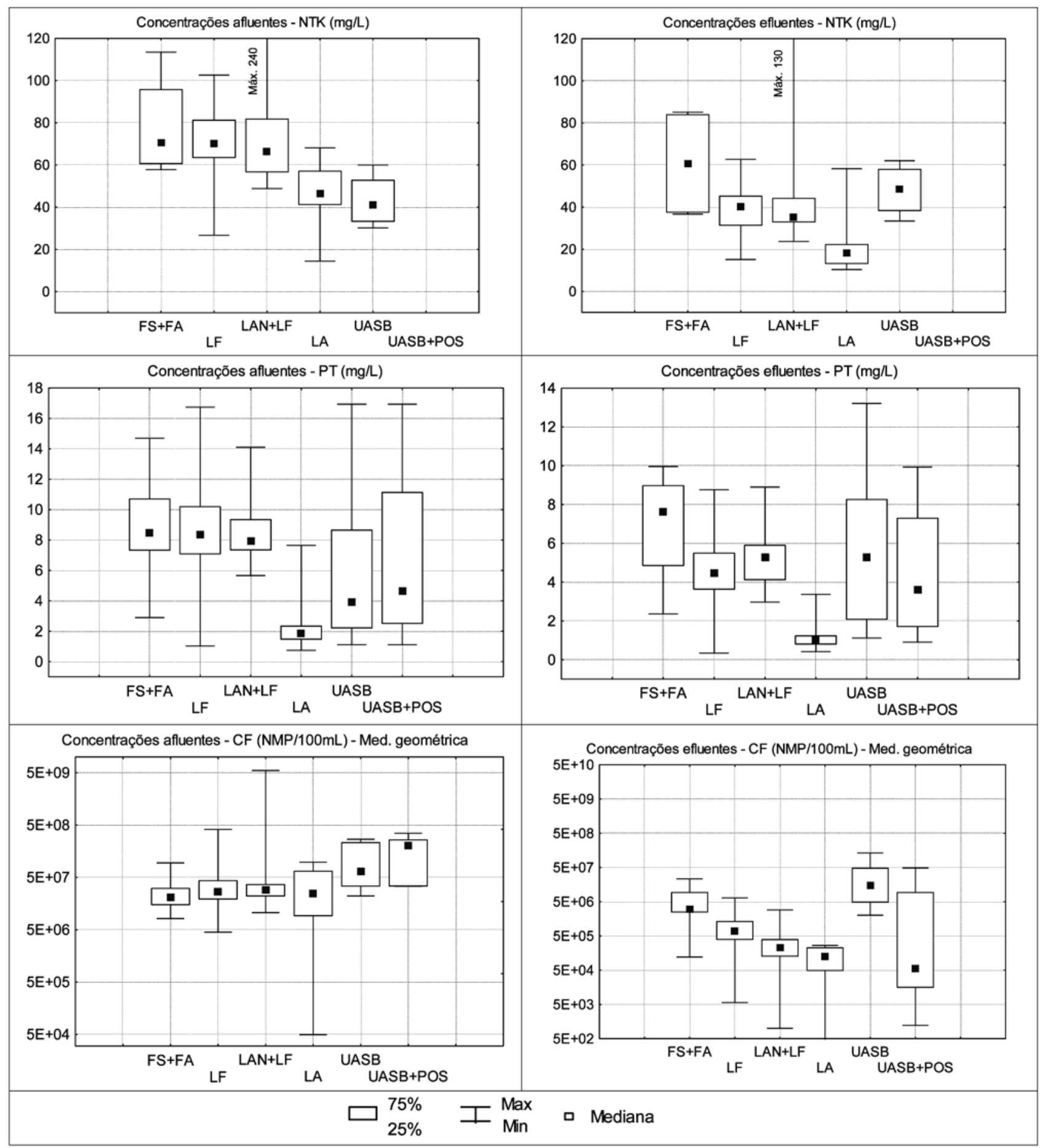

Figura I - Concentrações médias afluentes e efluentes dos diversos constituintes (Cont.) 
riam necessárias investigações mais específicas para confirmação destas suposições.

$\mathrm{O}$ percentual de ETEs que apresentaram concentrações médias de DQO, SST e NTK acima dos valores típicos também foi elevado. Para PT o percentual não foi tão expressivo, e para CF as concentrações estiveram quase todas dentro da faixa usual reportada pela literatura (que é bastante ampla).

\section{Comparação entre as concentrações efluentes observadas e reportadas na literatura}

As concentrações efluentes observadas para os constituintes mostraram-se, também, mais elevadas do que o usual, para os diversos processos de tratamento. A título de comparação, foram extraídos alguns dados de uma tabela apresentada em von Sperling (2005) para 37 diferentes tecnologias de tratamento de esgotos, onde constam valores mínimos e máximos esperados para concentraçōes efluentes de esgotos domésticos. Esses valores típicos esperados para as seis modalidades analisadas e as faixas efetivamente observadas para as ETEs em operação, considerando os percentis de $10 \%$ e $90 \%$, são apresentadas na Tabela 4.

Para o processo de lodos ativados (LA), que agrupou os sistemas convencional e aeração prolongada, foram considerados o menor e o maior valor para com- posição das faixas mínimas e máximas, respectivamente. No caso dos reatores UASB seguidos de pós-tratamento (UASB+POS), foram extraídas da tabela original as faixas esperadas para concentrações efluentes dos sistemas utilizados como pós-tratamentos.

Pela observação da Tabela 4, notase que, de uma maneira geral, houve uma grande diferença entre as faixas reportadas pela literatura como usuais e as efetivamente observadas, considerando todos os constituintes e todas as tecnologias de tratamento.

FS+FA. No caso das FS+FA, esta diferença foi muito expressiva considerando, principalmente, DBO, DQO e SST. Foram observadas concentrações efluentes de DBO e DQO até dez vezes maiores que os limites superiores das faixas de referência e de sete vezes para SST. Considerando os nutrientes, o desempenho ficou dentro do esperado para a modalidade, enquanto os resultados de concentraçōes efluentes de CF estiveram até melhores que as expectativas, apresentando cerca de $18 \%$ dos resultados abaixo do valor inferior da faixa reportada.

LF. As concentrações efluentes observadas para as lagoas facultativas (LF) mostraram-se, também, bastante superiores aos valores tomados como referência. Para DBO, DQO e SST, as menores concentrações observadas estiveram acima do limite superior das faixas reportadas pela literatura como usuais. Já as concentra- ções de CF estiveram todas dentro ou abaixo das esperadas para a modalidade.

LAN+LF. Os sistemas australianos (LAN+LF) apresentaram resultados mais próximos aos reportados na literatura, considerando os constituintes DBO, NTK e PT. As concentrações efluentes de DQO e SST estiveram superiores aos valores de referência e 100\% dos resultados observados para CF estiveram abaixo do esperado (desempenho melhor que o reportado como usual).

LA. A modalidade LA mostrou um bom ajuste às faixas reportadas para quase todos os constituintes. Em alguns casos o desempenho apresentado foi superior ao esperado, sendo observadas concentrações efluentes médias de NTK, PT e CF ainda menores que as consideradas usuais para modalidade.

UASB. Os reatores UASB operando isoladamente apresentaram um desempenho um pouco aquém do reportado pela literatura, considerando as concentraçôes de DQO, dos nutrientes e de CF.

UASB+POS. Quando os reatores UASB operaram seguidos por algum tipo de pós-tratamento (UASB+POS), os resultados obtidos foram os que melhor se ajustaram aos valores reportados como usuais, considerando todos os constituintes.

Para facilitar a visualização das diferenças entre as concentraçōes típicas expressas na literatura e aquelas efetivamente observadas, foram elaborados os gráficos

Tabela 4 - Concentrações efluentes usuais reportadas e concentrações médias observadas

\begin{tabular}{|c|c|c|c|c|c|c|c|}
\hline Constituinte & Faixas & $\mathrm{FS}+\mathrm{FA}$ & $\mathrm{LF}$ & $\mathrm{LAN}+\mathrm{LF}$ & LA & UASB & $\mathrm{UASB}+\mathrm{POS}$ \\
\hline \multirow{2}{*}{$\begin{array}{c}\mathrm{DBO} \\
(\mathrm{mg} / \mathrm{L})\end{array}$} & Literatura $^{(1)}$ & 40 a 80 & 50 a 80 & 50 a 80 & 10 a 40 & 70 a 100 & 20 a 80 \\
\hline & Observadas ${ }^{(2)}$ & 74 a 575 & 86 a 176 & 54 a 133 & 16 a 58 & 67 a 129 & 13 a 63 \\
\hline \multirow{2}{*}{$\begin{array}{l}\text { DQO } \\
(\mathrm{mgL})\end{array}$} & Literatura & 100 a 200 & 120 a 200 & 120 a 200 & 30 a 120 & 180 a 270 & 60 a 200 \\
\hline & Observadas & 159 a 1134 & 342 a 676 & 213 a 421 & 35 a 188 & 147 a 344 & 61 a 219 \\
\hline \multirow{2}{*}{$\begin{array}{c}\text { SST } \\
(\mathrm{mg} / \mathrm{L})\end{array}$} & Literatura & 30 a 60 & 60 a 90 & 60 a 90 & 20 a 40 & 60 a 100 & 10 a 90 \\
\hline & Observadas & 53 a 290 & 132 a 343 & 80 a 236 & 13 a 130 & 49 a 137 & 17 a 85 \\
\hline \multirow{2}{*}{$\begin{array}{l}\mathrm{NT}^{(3)} \\
(\mathrm{mg} / \mathrm{L})\end{array}$} & Literatura & $>20$ & $>20$ & $>20$ & $>20$ & $>20$ & $15 a>30$ \\
\hline & Observadas & 37 a 84 & 25 a 48 & 26 a 69 & 12 a 33 & 36 a 60 & - \\
\hline \multirow{2}{*}{$\begin{array}{c}\mathrm{PT} \\
(\mathrm{mg} / \mathrm{L})\end{array}$} & Literatura & $>4$ & $>4$ & $>4$ & $>4$ & $>4$ & $1 a>4$ \\
\hline & Observadas & 4 a 9 & 2 a 7 & 4 a 8 & 1 a 2 & 2 a 11 & 1 a 8 \\
\hline \multirow{2}{*}{$\begin{array}{c}\text { CF } \\
(\text { org/100mL) }\end{array}$} & Literatura & $10^{6}$ a $10^{7}$ & $10^{6}$ a $10^{7}$ & $10^{6}$ a $10^{7}$ & $10^{6}$ a $10^{7}$ & $10^{6}$ a $10^{7}$ & $10^{2}$ a $10^{7}$ \\
\hline & Observadas & $4 \times 10^{5}$ a $1 \times 10^{7}$ & $2 \times 10^{5}$ a $2 \times 10^{6}$ & $7 \times 10^{4}$ a $1 \times 10^{6}$ & $3 \times 10^{4}$ a $3 \times 10^{5}$ & $4 \times 10^{6}$ a $7 \times 10^{7}$ & $9 \times 10^{3}$ a $3 \times 10^{7}$ \\
\hline
\end{tabular}

(1) Faixa reportada pela literatura: adaptada de von Sperling (2005);

(2) Faixas observadas: foram utilizados os percentis 10\% (valor mínimo) e 90\% (valor máximo);

(3) Foram utilizados valores de NTK e NT. 
mostrados na Figura 2. Neles estão representadas as faixas reportadas na literatura, bem como os percentuais de ETEs que apresentaram concentraçôes efluentes médias abaixo das faixas mínimas, dentro das faixas e acima das faixas máximas descritas na literatura para cada tecnologia e constituinte. A Tabela 4 apresenta apenas os valores mínimos para NT (20 mg/L) e PT ( $4 \mathrm{mg} / \mathrm{L})$, que foram tomados como referência para cálculo do percentual de ETEs com concentrações abaixo ou acima destes valores. Apenas os reatores $\mathrm{UASB}+\mathrm{POS}$ possuíam um intervalo específico para a categoria.
Como exemplo de interpretação dos gráficos apresentados na Figura 2, tomese o caso da DBO e da modalidade $\mathrm{FS}+\mathrm{FA}$.

FS+FA. Nesta modalidade, a faixa de concentraçóes efluentes reportada na literatura é de 40 a $80 \mathrm{mg} / \mathrm{L}$ (ver também Tabela 4). O sistema FS+FA apresentou $90 \%$ das ETEs com concentraçōes efluentes médias acima do valor máximo de $80 \mathrm{mg} / \mathrm{L}$ (portanto, pior desempenho que o esperado), $5 \%$ dentro da faixa de 40 a $80 \mathrm{mg} / \mathrm{L}$ e $5 \%$ abaixo do valor mínimo de $40 \mathrm{mg} / \mathrm{L}$ reportado na literatura como usual para esta tecnologia de tratamento. Esse desempenho aquém das expectativas foi observado também para os outros constituintes analisados, exceção feita para os $\mathrm{CF}$, que apresentaram $82 \%$ das ETEs com desempenho acima ou dentro do esperado.

LF, LAN+LF, UASB. As concentraçōes efluentes observadas para estes sistemas estiveram acima dos valores de referência (pior desempenho) ao se considerar praticamente todos os constituintes. Nesse caso, também, a única exceção foi observada para as concentraçôes efluentes médias de coliformes fecais, que se mostraram inferiores aos valores reportados.
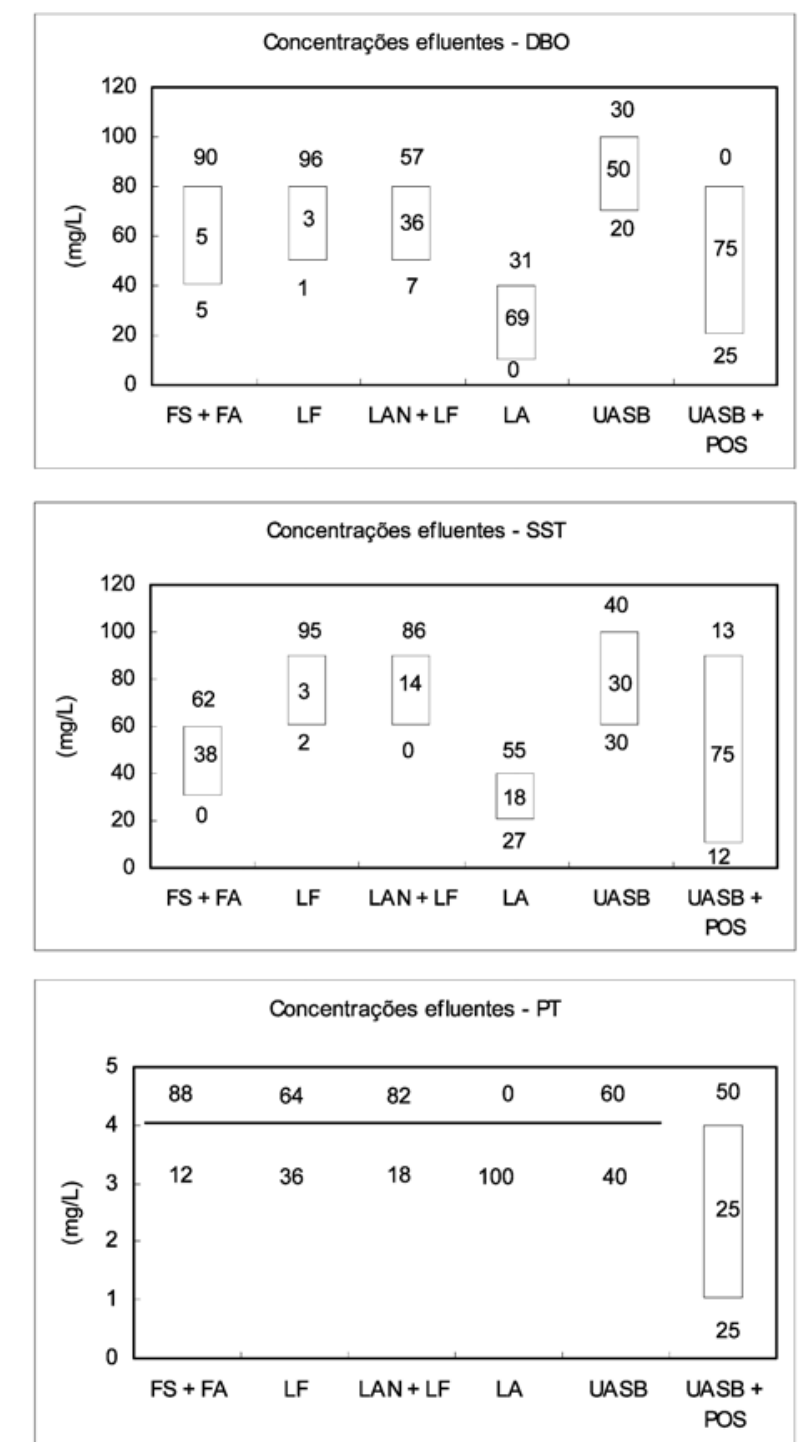
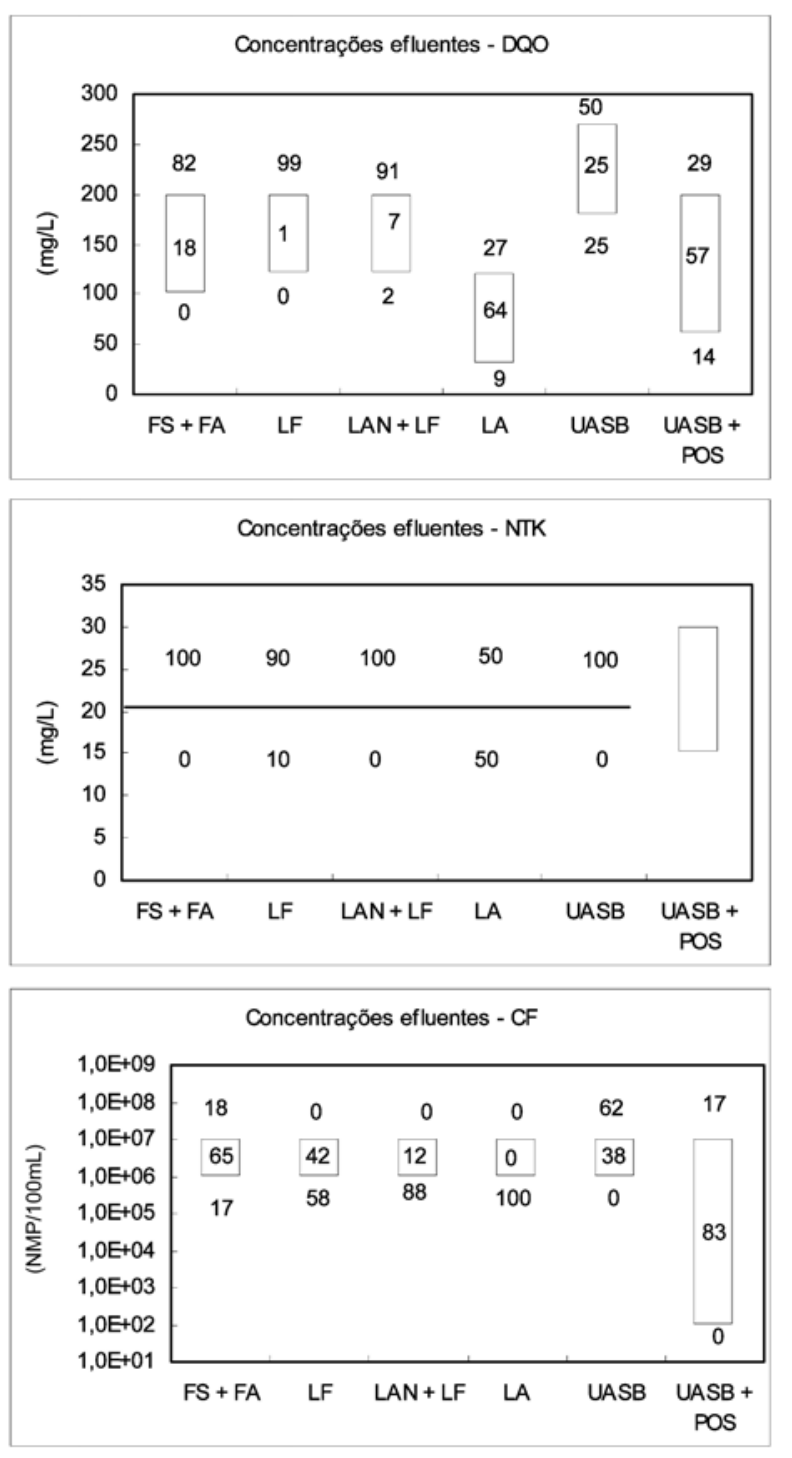

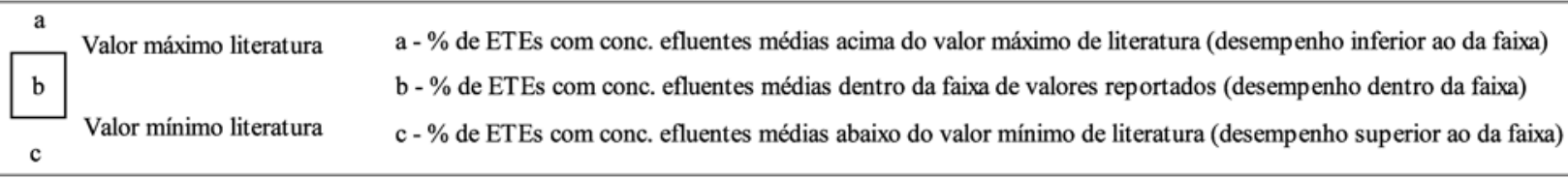

Figura 2 - Percentual de ETEs com concentrações efluentes médias abaixo, dentro ou acima das faixas de referência reportadas na literatura 
LA. A modalidade de LA mostrou concentraçóes efluentes de DBO e DQO mais ajustadas aos valores tomados como referência de desempenho. Esse comportamento não foi observado, no entanto, para os constituintes SST (desempenho inferior ao esperado), nutrientes e CF (desempenho superior às faixas reportadas).

UASB+POS. Os reatores UASB+POS mostraram os melhores ajustes aos valores reportados, considerando praticamente todos os constituintes. Para essa modalidade não foram disponibilizados dados de concentração efluente de NT ou NTK.

Ressalta-se que as concentrações observadas de CF efluente mostraram-se menores que as esperadas para quase todas as tecnologias de tratamento. Chamam a atenção, principalmente, os bons desempenhos apresentados pelas modalidades LA, LF e LAN+LF. As concentrações efluentes dos nutrientes estiveram acima dos valores máximos das faixas para a maioria absoluta dos processos. Este fraco desempenho já era esperado, já que nenhuma das modalidades analisadas foi projetada objetivando a remoção de nitrogênio ou fósforo. No entanto, o bom desempenho apresentado pelo processo de lodos ativados, considerando o PT, surpreende, chegando a apresentar 100\% das ETEs com concentraçóes efluentes inferiores ao esperado.

\section{Comparação entre as eficiências de remoção observadas e reportadas na literatura}

Foram investigadas, ainda, as eficiências de remoção das cargas poluidoras afluentes das seis modalidades de tratamento. As estatísticas descritivas são apresentadas na Figura 3. As eficiências de remoção foram, também, comparadas com as reportadas pela literatura para os diversos processos de tratamento. Parte dos dados citados por von Sperling (2005) para as diferentes tecnologias de tratamento de esgotos, referentes aos valores mínimos e máximos esperados para eficiências de remoção, foram utilizados para elaboração da Tabela 5. São apresentadas, também, as faixas de eficiências de remoção dos seis constituintes que foram efetivamente observadas para as ETEs em operação.

A Figura 4 apresenta os gráficos que mostram os percentuais de ETEs, considerando cada modalidade de tratamento, que apresentaram eficiências de remoção acima, dentro ou abaixo das faixas reportadas pela literatura.

As Figuras 3 e 4 permitiram algumas conclusóes, considerando as seis modalidades de tratamento:

FS+FA. Observou-se um alto percentual de eficiências médias de remoção menores que os valores mínimos esperados para a modalidade FS+FA. Esse desempenho aquém do esperado foi observado para todos os constituintes analisados, excetuando-se remoção de CF.

LF. No caso das lagoas facultativas (LF), um percentual bastante elevado de ETEs mostrou eficiências de remoção de DQO (76\%), SST (91\%) e NTK (90\%) abaixo da faixa mínima de referência reportada. No entanto, boas eficiências de remoção de PT e CF foram observadas.

LAN+LF. Os sistemas australianos (LAN+LF) mostraram um bom desempenho em termos de remoção de DBO, DQO, PT e CF, apresentando um percentual expressivo de ETEs com eficiências acima do limite superior das faixa consideradas.

LA. O processo de lodos ativados foi o que apresentou as maiores eficiências de remoção da matéria orgânica. No entanto, a aderência às faixas da literatura, considerando as remoções de DBO e SST, ficou abaixo do que seria esperado. Isto pode ser parcialmente explicado pelas baixas concentraçôes afluentes ao sistema LA, considerando todos os constituintes (conforme apresentado na Tabela 2), o que dificulta a obtenção de elevadas eficiências de remoção.

UASB. Os reatores UASB mostraram uma boa eficiência de remoção de $\mathrm{DBO}$ e DQO e um fraco desempenho

Tabela 5 - Eficiências típicas reportadas na literatura e eficiências efetivamente observadas

\begin{tabular}{cccccccc}
\hline Constituinte & Faixas & FS+FA & LF & LAN+LF & LA & UASB & UASB+POS \\
\hline DBO (\%) & Literatura $^{(1)}$ & 80 a 85 & 75 a 85 & 75 a 85 & 85 a 97 & 60 a 75 & 75 a 93 \\
& Reais ${ }^{(2)}$ & 36 a 82 & 65 a 84 & 73 a 88 & 74 a 96 & 65 a 79 & 85 a 92 \\
DQO (\%) & Literatura & 70 a 80 & 65 a 80 & 65 a 80 & 80 a 93 & 55 a 70 & 65 a 90 \\
& Reais & 18 a 78 & 40 a 72 & 65 a 78 & 62 a 93 & 44 a 77 & 64 a 86 \\
SST (\%) & Literatura & 80 a 90 & 70 a 80 & 70 a 80 & 87 a 93 & 65 a 80 & 70 a 97 \\
& Reais & 39 a 86 & 23 a 69 & 35 a 78 & 53 a 95 & 57 a 81 & 71 a 92 \\
NT (\%) & Literatura & $<60$ & $<60$ & $<60$ & $<60$ & $<60$ & $<30$ a 65 \\
& Reais & 3 a 39 & 34 a 58 & 26 a 60 & 20 a 78 & -19 a -6 & - \\
PT (\%) & Literatura & $<35$ & $<35$ & $<35$ & $<35$ & $<35$ & $<35$ a 88 \\
& Reais & -27 a 45 & 32 a 63 & 20 a 50 & 35 a 55 & -21 a 16 & -8 a 49 \\
CF (unid. log & Literatura & 0,5 a 1,5 & 1 a 2 & 1 a 2 & 1 a 2 & 0,5 a 1,5 & 1 a 5 \\
removida) & Reais & 0,5 a 1,8 & 1,0 a 2,4 & 1,6 a 3,0 & 1,4 a 2,8 & 0 a 1,3 & 0,8 a 5,2 \\
\hline
\end{tabular}

(1) Faixa reportada pela literatura: adaptada de von Sperling (2005);

(2) Faixas observadas: foram utilizados os percentis $10 \%$ (valor mínimo) e $90 \%$ (valor máximo). 


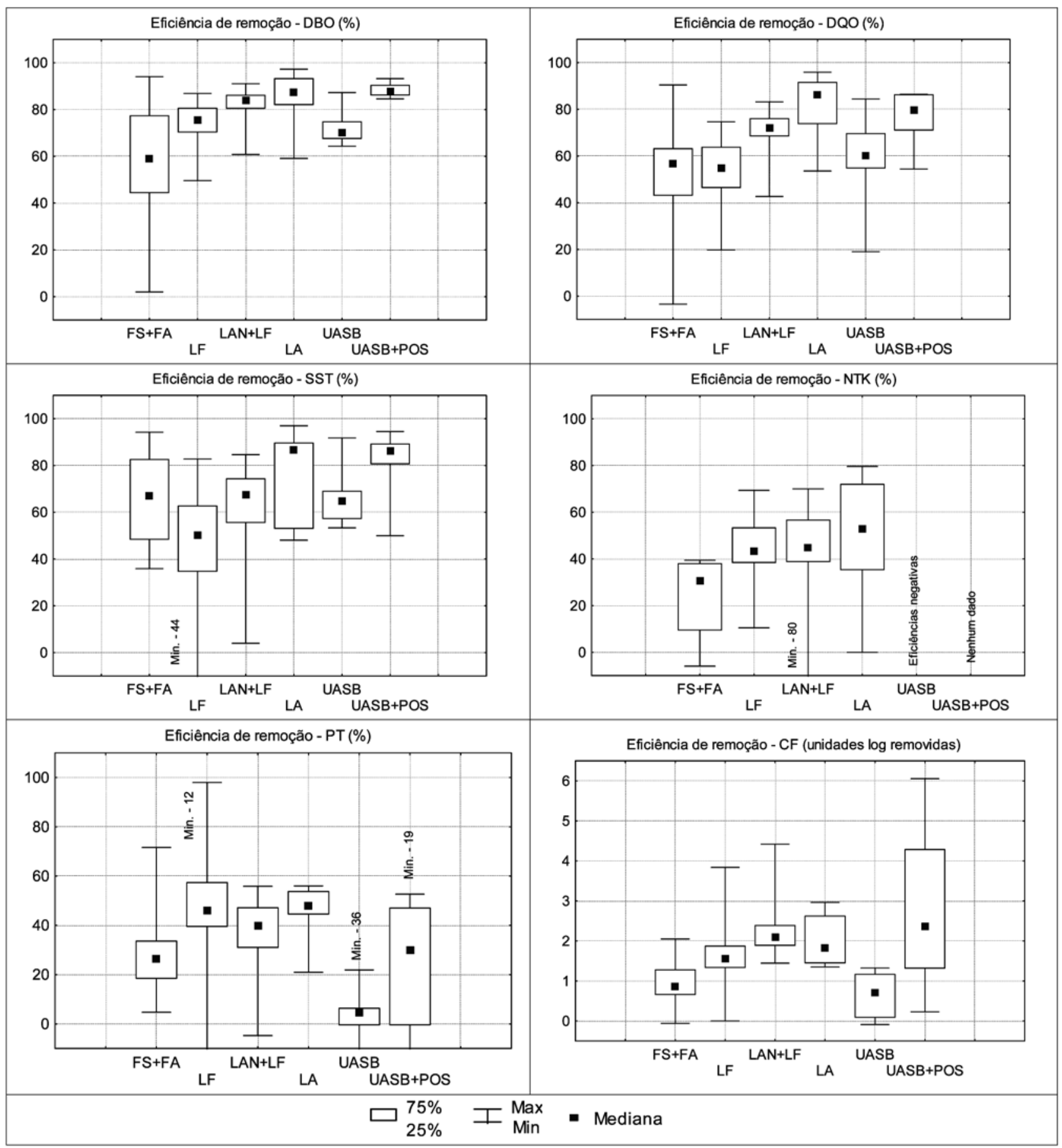

Figura 3 - Eficiências médias de remoção dos diversos constituintes 

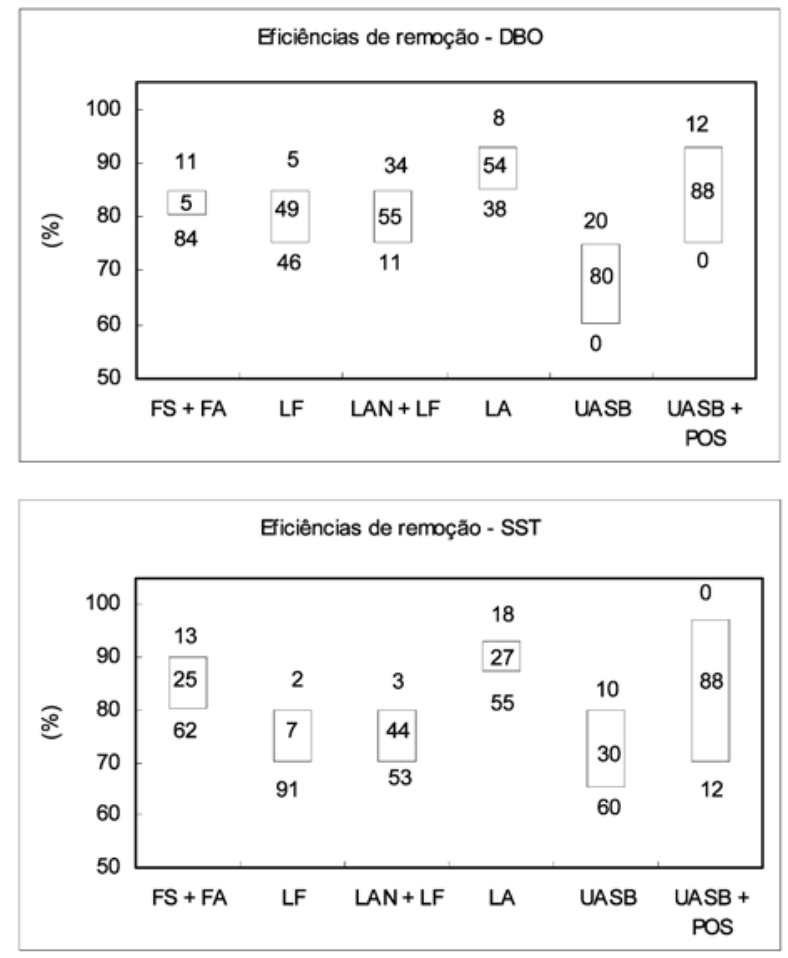

Eficiências de remoçăo - PT

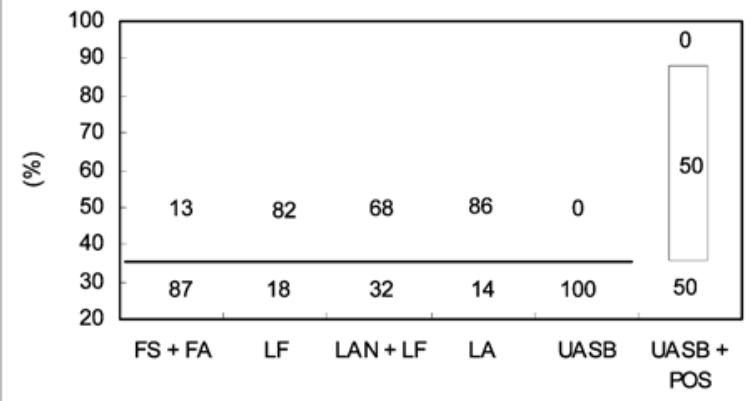

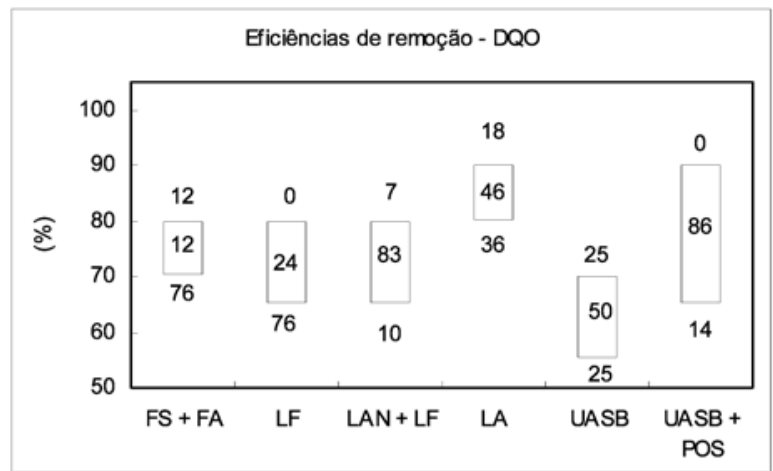
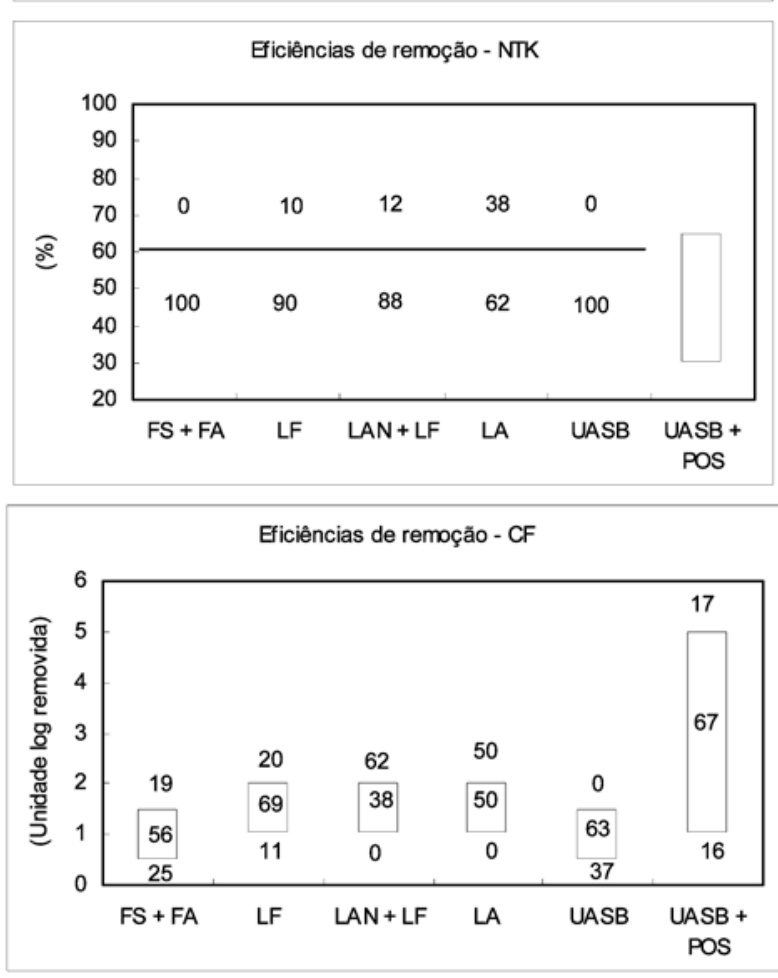

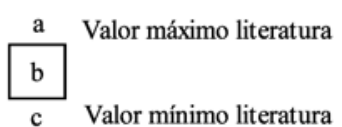

a - \% de ETEs com eficiências médias acima do valor máximo de literatura (desempenho superior ao da faixa)

b - \% de ETEs com eficiências médias dentro da faixa de valores reportados (desempenho dentro da faixa)

c - \% de ETEs com eficiências médias abaixo do valor mínimo de literatura (desempenho inferior ao da faixa)

\section{Figura 4 - Percentual de ETEs com eficiências médias de remoção abaixo, dentro ou acima das faixas de referência reportadas na literatura}

em relação às faixas de referência reportadas na literatura, considerando SST e nutrientes.

UASB+POS. O desempenho obtido pelos reatores UASB seguidos de póstratamento foi o que melhor se ajustou aos dados de literatura, considerando praticamente todos os constituintes. No entanto, as faixas da literatura são mais amplas, devido à diversidade de possíveis sistemas de pós-tratamento.

\section{CONCLUSÕES}

Os resultados obtidos mostraram que, especialmente nas ETES dos pro- cessos FS+FA, LF e LAN+LF, os esgotos afluentes apresentaram uma concentração mais elevada do que usualmente expresso na literatura. Algumas possibilidades foram aventadas para justificar esta situação, tais como, contribuiçôes industriais não relatadas pelas ETEs, tipo de amostragem praticado, baixo consumo per capita de água e, ainda, existência de menores coeficientes de retorno. No entanto, seriam necessárias investigações mais específicas e estudos mais aprofundados para confirmação destas suposiçōes.

Foi observada uma grande variabilidade nas concentraçôes afluentes e efluentes, considerando todos os constituintes analisados e todas as modalidades de tratamento.

O percentual de ETEs da modalidade FS+FA que apresentou um desempenho abaixo do esperado foi muito elevado, considerando praticamente todos os constituintes. Apenas para CF este comportamento não foi verificado. No caso das lagoas facultativas (LF), um percentual bastante elevado de ETEs mostrou eficiências de remoção de DQO, SST e NTK aquém das expectativas. No entanto, boas eficiências de remoção de PT e CF foram observadas. Os sistemas australianos (LAN+LF) mostraram um bom 
desempenho em termos de remoção de DBO, DQO, PT e CF, apresentando um percentual expressivo de ETEs com eficiências dentro e até acima do esperado. O desempenho apresentado pelo processo de lodos ativados (LA), considerando a remoção de matéria orgânica, foi o mais elevado dentre os sistemas avaliados, embora tenha se situado abaixo dos altos níveis que seriam esperados. Os reatores UASB mostraram uma boa eficiência de remoção de $\mathrm{DBO}$, DQO e CF e um fraco desempenho considerando SST e nutrientes, considerando as faixas de referência reportadas na literatura. $\mathrm{O}$ desempenho obtido pelos reatores UASB seguidos de pós-tratamento foi o que melhor se ajustou aos dados de literatura, considerando praticamente todos os constituintes.

Sabe-se que as condiçôes de carga hidráulica e orgânica aplicada, do nível de operação, de aspectos hidráulicos e de fatores de projeto e de construção exercem uma grande influência no desempenho das ETEs. Estes aspectos não foram considerados no presente trabalho, que procurou apenas apresentar um diagnóstico da realidade do tratamento de esgotos no Brasil, considerando as principais tecnologias de tratamento, em termos da qualidade do seu efluente. Os resultados apresentados retratam a realidade ora vivenciada, mas não o potencial de cada uma das tecnologias investigadas, que podem atingir desempenhos superiores aos apresentados. Portanto, para buscar elucidar os fatores que conduziram a um melhor ou pior desempenho, e também para evitar que dúvidas sejam lançadas sobre a capacidade de alguns dos processos de tratamento analisados, efetuou-se um estudo de avaliação da influência de fatores de projeto e de operação no desempenho das estaçóes de tratamento de esgotos. Este estudo encontra-se apresentado na Parte 2 do presente trabalho (Oliveira e von Sperling, 2005).

\section{AGRADECIMENTOS}

USP, FINEP, SABESP, COPASA, FEAM, Fapemig e CNPq e prestadores de serviços municipais.

\section{REFERÊNCIAS}

ANDRADE NETO, C.O. Sistemas simples para tratamento de esgotos sanitários. Experiência brasileira. ABES. 302 p. 1997.

BERTHOUEX, P.M., HUNTER, W.G. How to construct reference distributions to evaluate treatment plant effluent quality. JWPCF, v. 55, n. 12 , p. $1417-1424,1983$.

CAMPOS, J.R. (coordenador). Tratamento de esgotos sanitários por processo anaeróbio e disposição controlada no solo. PROSAB/FINEP, Rio de Janeiro, 435 p. 1999.

CHERNICHARO, C.A.L. (coordenador) Póstratamento de efluentes de reatores anaeróbios. 1. ed. Belo Horizonte: FINEP/PROSAB, c. 7. p. 377-454. 2001.

DEAN, R.B., FORSYTHE, S.L. Estimating the reliability of advanced waste treatment. Part 1 and 2, Water \& Sewage Works, June, 1976 a and $1976 \mathrm{~b}$.

NIKU, S. et al. Performance of activated sludge process: reliability, stability and variability. Environmental Protection Agency, EPA Grant $\mathrm{N}^{\circ}$ R805097-01, pp. 1 - 124. 1981b.

OLIVEIRA, S.M.A.C.; VON SPERLING, M. Avaliação de 166 ETEs em operação no país, compreendendo diversas tecnologias. Parte 2: Influência de fatores de projeto e operação. Engenharia Sanitária e Ambiental (ABES), 2005.

SMITH, E.P. et al. Statistical assessment of violations of water quality standards under Section 303 (d) of the Clean Water Act. Environmental Science \& Technology, v. 35, n. 3, p.606612, 2001.

VAUGHAN, W.J. and RUSSELL, C.S. Monitoring point sources of pollution: answers and more questions from statistical quality control. The American Statistician, v. 37, Issue 4, Part 2: Proceedings of the Seventh Symposium on Statistics and the Environment, p. 476 - 487. Nov., 1983.

VON SPERLING, M. Associação entre a legislação brasileira de qualidade da água (Resolução
CONAMA 20/86) e a seleçāo de processos de tratamento de esgotos. Engenharia Sanitária e Ambiental (ABES), Ano 3, v 3, n. 1/2, p. 67-73, Jan/Jun 1998.

VON SPERLING, M. Princípios do tratamento biológico de águas residuárias. Vol. 1. Introdução à qualidade das águas e ao tratamento de esgotos. DESA - UFMG. $3^{a}$ ed. 456 p. 2005.

VON SPERLING, M., CHERNICHARO, C.A.L. A comparison between wastewater treatment processes in terms of compliance with effluent quality criteria standards. In: XXVII CONGRESO INTERAMERICANO DE INGENIERIA SANITARIA Y AMBIENTAL, Porto Alegre. Anais, Porto Alegre: AIDIS, 2000.

VON SPERLING, M., CHERNICHARO, C.A.L. Urban wastewater treatment technologies and the implementation of discharge standards in developing countries. Urban Water, v. 4. p. 105-114, 2002.

Endereço para correspondência:
Marcos von Sperling
Departamento de Engenharia
Sanitária e Ambiental - DESA
Universidade Federal de Minas
Gerais
Av. Contorno, 842 - $7^{\circ}$ andar
30 I I 0-060 Belo Horizonte - MG-
Brasil
Tel: (3I) 3238-1935
Fax: (3I) 3238-I879
E-mail:marcos@desa.ufmg.br

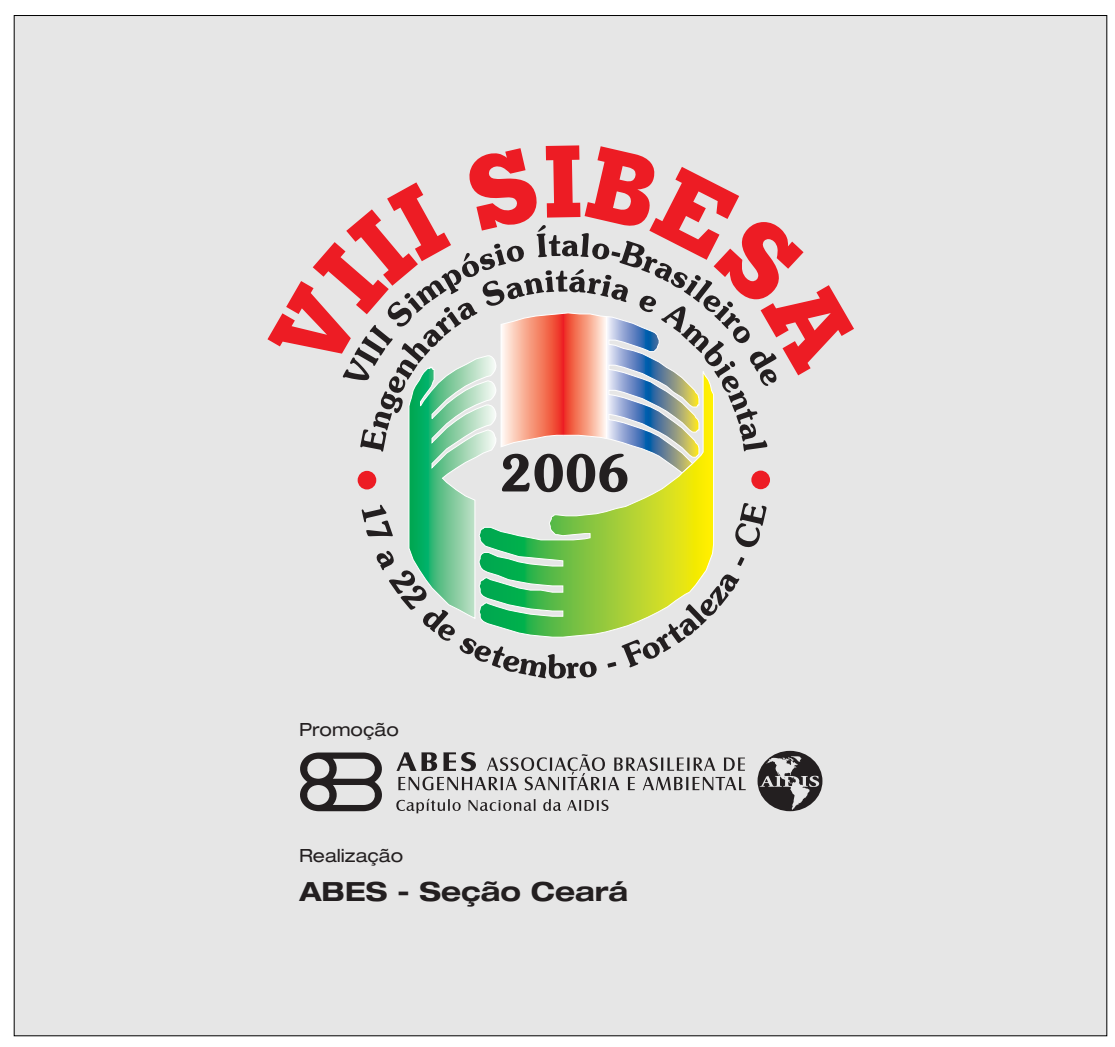

